lungs (spirography), kidneys (concentration function, glomerular filtration rate, daily microalbuminuria), lipid spectrum and blood coagulation. Deviations from standard age-gender normal values were taken into account. It was used statistical methods for identifying relationships (stepwise multiple regression). As the dependent value the number of detected changes in the state of internal organs and systems is taken. The independent variables included standard clinical and laboratory parameters, the age of the onset of $\mathrm{JIA}$, the total duration of the disease, the characteristic of the articular syndrome, activity indicators (C-reactive protein, RF and ANA titers, JADAS27), as well as the term for the appointment of basic therapy relative to the debut of the disease, the nature of the dosage methotrexate ( $\mathrm{mg} / \mathrm{m} 2$ / week), the duration of its use at the time of examination.

Results: Based on the analysis and the obtained regression models for the formation of extra-articular lesions in children with various JIA options, it was found that the leading factors in the formation of comorbidity are the duration of the disease $(p=0.01)$, VAS results (including the child, parents and the physician) ( $p$ $=0.01)$, the dose of methotrexate $(p=0.01)$.

In the polyarticular variant, the body mass index $(p=0.02)$, erythrocyte content ( $p=0.007$ ) were significant for the formation of comorbid states and should be distinguished.

The overall duration of the disease $(p=0.02)$, the low age of initiation of therapy $(p=0.05)$, VAS of child $(p=0.003)$, CRP $(p=0.007)$, dose of methotrexate ( $p$ $=0.001$ ) had the greatest significance level among the clinical and laboratory indicators included in the regression model of the formation of comorbidity with the oligoarticular variant.

A features of the prognostic model for the formation of organ lesions and metabolic disorders in uveitis-associated arthritis were GCS therapy presence ( $p$ $<0.001$ ), patient age ( $p<0.001)$, JADAS27 $(p<0.001)$ and methotrexate dose $(\mathrm{p}<0.001)$

Conclusion: Thus, in children with JIA, the formation of comorbid pathology is associated with a lower age of JIA debut in oligoarthritis and uveitis-associated arthritis, low body weight in poly- and oligoarticular JIA variants, an increase in the duration of the disease in the polyarticular variant, and the level of activity of the process, respectively, JADAS $27(p<0.01)$ and VAS. Prognostically unfavorable for the formation of pathological changes in the internal organs and homeostasis are anemia, high white blood cell count and ESR level.

Disclosure of Interests: None declared

DOI: 10.1136/annrheumdis-2020-eular.5922

\section{FRI0472 DIAGNOSIS AND MANAGEMENT OF ADENOSINE DEAMINASE 2 DEFICIENCY CHILDREN: THE EXPERIENCE FROM CHINA}

W. Wang ${ }^{1}$, T. Zhang ${ }^{1}$, L. Wang ${ }^{1}$, H. Song ${ }^{1} .{ }^{1}$ Chinese Academy of Medical Sciences, Pediatrics, Beijing, China

Background: Adenosine deaminase 2 deficiency (DADA2) is a rare antoinflammatory disease caused by mutations in ADA2 gene, few Chinese cases have been reported.

Objectives: To describe and compare the clinical features, genotypes, and treatments of Chinese DADA2 patients and foreign cases.

Methods: Primary immunodeficiency disease Panel or Whole Exome Sequencing was performed to suspected subjects, and assays for adenosine deaminase 2(ADA2) enzyme activity were also carried out to them and their parents. Case reports of Chinese and foreign patients with DADA2 were searched from PubMed and Chinese domestic databases.

Results: Seven unrelated DADA2 children from China were included in our study, 5 were identified at Peking union medical college hospital and 2 had been reported previously ( 1 on PubMed and 1 in Chinese literatures). 14 mutations in ADA2 were identified, and 9 of which have not been found in other countries. Four children receiving enzymatic analysis had lower ADA2 enzyme activity compared to their parents. Phenotypic manifestations included fever, skin symptoms, vasculitis, neurologic involvement, et al. The treatments varying from steroids, immunosuppressants, and tocilizumab, anti-TNF therapy and hematopoietic stem cell transplantation (HSCT) were effective depending on different phenotype and severity.

Conclusion: This study includes the biggest number of Chinese DADA2 patients at present. We recommend combination of enzymatic analysis with gene screening to confirm the diagnosis. Genotypes of patients from China were some different, the clinical manifestations were similar. We suggest anti-TNF therapy may not be necessary for mild case and HSCT should be considered even without hematological phenotype.

References:

[1] Zhou Q, Yang D, Ombrello AK, Zavialov AV, Toro C, Zavialov AV, et al. Early-onset stroke and vasculopathy associated with mutations in ADA2. N Engl J Med. 2014;370:911-920.
[2] Meyts I, Aksentijevich I. Deficiency of Adenosine Deaminase 2 (DADA2): Updates on the Phenotype, Genetics, Pathogenesis, and Treatment. J Clin Immunol. 2018;38:569-578.

[3] Wang XN, Zhou ZX, Li SN, Lai JM, Su GX, Kang M, et al. A case report of DADA2. Chin J Rheumatol. 2019;23:476-478.

[4] Liu L, Wang W, Wang Y, Hou J, Ying W, Hui X, et al. A Chinese DADA2 patient: report of two novel mutations and successful HSCT. Immunogenetics. 2019;71:299-305.

Disclosure of Interests: None declared

DOI: 10.1136/annrheumdis-2020-eular.3481

\section{FRIDAY, 05 JUNE 2020}

\section{Other orphan diseases}

\section{FRI0473 ASSESSMENT OF FEMORAL VEIN WALL THICKNESS WITH DOPPLER US AS A DIAGNOSTIC TOOL FOR BEHCET'S DISEASE}

F. Alibaz-Oner ${ }^{1}$, R. Ergelen ${ }^{1}$, Y. Yıldız ${ }^{1}$, A. Yazici ${ }^{2}$, M. Aldag ${ }^{3}$, A. Cefle $^{2}$, B. Artim-Esen ${ }^{4}$, G. Mumcu ${ }^{5}$, T. Ergun ${ }^{6}$, H. Direskeneli ${ }^{6} .{ }^{1}$ Marmara University, School of Medicine, Rheumatology, İstanbul, Turkey; ${ }^{2}$ Kocaeli University, School of Medicine, Kocaeli, Turkey; ${ }^{3}$ Bahçeşehir University, Cardiovascular Surgery Department, Istanbul, Turkey; ${ }^{4}$ Istanbul University, Istanbul Medical School, Istanbul, Turkey; ${ }^{5}$ Marmara University, Health Science Faculty, Istanbul, Turkey; ${ }^{6}$ Marmara University, School of Medicine, Istanbul, Turkey

Background: Diagnosing Behcet's disease(BD) is a challenge, especially in countries with a low prevalence. International Study Group Criteria, accepted to as diagnostic, has low sensitivity, especially in early cases when major organ involvement such as uveitis or deep vein thrombosis(DVT) presents alone. We recently published a controlled study of assessing venous wall thickness(VWT) as a surrogate marker of venous disease in BD with ultrasound(US) and observed a very sensitive and specific VWT in male BD patients. The common femoral vein(CFV) thickness measurement, as the primary site of US with the cut-off values $>0.48-0.49 \mathrm{~mm}$, had a high area under the receiver operating characteristic curve( $>0.8)$ with sensitivity and specificity of around $80 \%(1)$

Objectives: In this study, we aimed to investigate the diagnostic performance of CVF thickness measurement in BD including females comparing with multiple control disease groups.

Methods: One hundred-ten patients with $\mathrm{BD}, 47$ healthy controls $(\mathrm{HC}), 21$ patients with systemic vasculitides, 28 patients with venous insufficiency,29 patients with antiphospholipid syndrome (APS) having DVT history, were included the study. Bilateral CFV thickness was measured with US by an experienced radiologist blinded to cases(Figure 1).

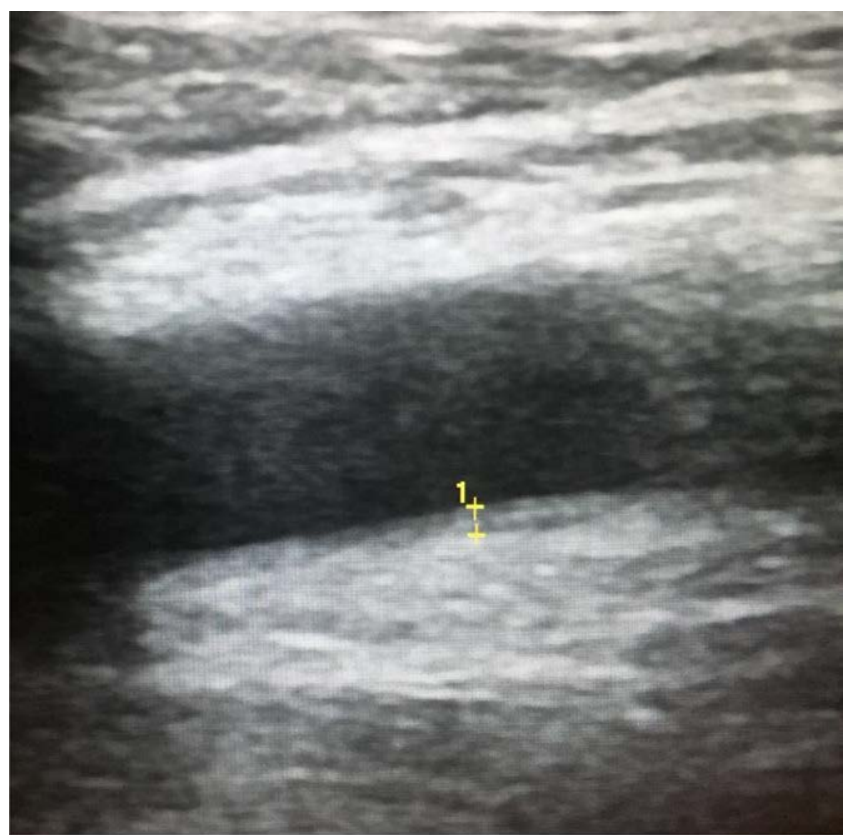

Figure 1. Measurement of common femoral vein thickness 